\title{
Total antioxidant status and 8-hydroxy-2'-deoxyguanosine levels in gingival and peripheral blood of periodontitis patients
}

\author{
Tomasz Konopka ${ }^{1}$, Katarzyna Król ${ }^{2}$, Wacław Kopeć ${ }^{3}$ and Hanna Gerber ${ }^{4}$ \\ ${ }^{1}$ Department of Oral Pathology, Wrocław Medical University, Wrocław, Poland \\ ${ }^{2}$ Department of General Dentistry, Pomeranian Medical University, Szczecin, Poland \\ ${ }^{3}$ Immunological Laboratory of Nephrology Clinic, Wrocław Medical University, Wrocław, Poland \\ ${ }^{4}$ Department of Maxillofacial Surgery, Wrocław Medical University, Wrocław, Poland
}

Received: 2007.03.07, Accepted: 2007.10.25, Published online first: 2007.12.03

\begin{abstract}
Introduction: The aim of this study was to determine 8-OHdG concentration as a biomarker of oxidant-induced DNA damage and to assess total antioxidant status (TAS) in gingival and peripheral blood during periodontal lesion.

Materials and Methods: The study included 56 untreated periodontitis patients ( 26 with aggressive periodontitis, and 30 with chronic periodontitis (CP). The control group consisted of 25 healthy volunteers without pathological changes in the periodontium. Competitive ELISA was used to measure 8-OHdG. A colorimetric method based on the reduction of ABTS ${ }^{\circ+}$ radical cation generation was used to measure TAS.

Results: Significantly higher 8-OHdG concentrations were detected in the gingival blood in both groups of patients with periodontitis than in the control group. Subjects with CP had significantly decreased TAS levels in the gingival blood compared with the control group. A significantly decreased TAS level in the peripheral blood in both patient groups compared with the control group was found. Significant positive correlation between TAS levels in venous and gingival blood in all the periodontitis patients and in the CP group was observed.

Conclusions: The oxidative burst in periodontitis may lead to significant local damage to nucleic acids. The significantly decreased TAS level in the gingival blood of CP patients compared with the healthy subjects suggests the possibility of a significant decrease in local antioxidant system capacity during the course of periodontitis. The decreased TAS level in the peripheral blood in the group of all patients with periodontitis may be one of the pathogenic mechanisms underlying the links between periodontal disease and several systemic diseases for which periodontitis is regarded as a independent risk factor.
\end{abstract}

Key words: reactive oxygen species, 8-hydroxy-2'-deoxyguanosine, total antioxidant status, oxidative stress, periodontitis.

Abbreviations: 8-OHdG - 8-hydroxy-2'-deoxyguanosine, AgP - aggressive periodontitis, CP - chronic periodontitis, ELISA enzyme-linked immunosorbent assay, GCF - gingival crevicular fluid, PBI - papillary bleeding index, PD - periodontal pocket depth, PI - plaque index, ROS - reactive oxygen species, TAS - total antioxidant status.

Corresponding author: Tomasz Konopka, DMD Ph.D., Department of Oral Pathology, Wrocław Medical University, Krakowska 26, 50-425 Wrocław, Poland, tel./fax: +48 71 784-03-81, e-mail: tkonopka@stom.am.wroc.pl

\section{INTRODUCTION}

Reactive oxygen species (ROS) encompass a variety of partially reduced metabolites of oxygen. They are generated through a variety of processes, e.g. ionizing radiation, ultrasound, UV radiation, respiratory protein oxidation, activation of the mitochondrial respiratory chain, and from peroxisomes and enzymatic reactions. They are also produced in a "respiratory burst" from phagocytes. They act in phagosomes as agents toxic to microorganisms, fungi, parasites, and neoplasmic cells and are thus a decisive factor in phagocytes' intracellular killing mechanism [11, 36]. While most ROS have extremely short half-lives, they can cause substantial damage to tissue and cellular components, e.g. cellular phospholipids, nucleic acids, proteins, carbohydrates, and enzymes. Lipid peroxidation is initiated by the hydroxyl radical, which affects polyunsaturated fatty acids or membrane phospholipids [35]. Lipid hydroperoxides and aldehydes are formed in this way; as second 
messengers they lead to protein and DNA damage. The physical properties of cellular membrane are modified and the mitochondrial respiratory chain activity is decreased. The hydroxyl radical, in cooperation with metal ions bound to the DNA chain, reacts directly with nucleic acids, causing DNA damage and cracking, which may have mutagenic consequences $[6,10,14]$. Another effect of excess ROS on DNA is the activation of specific metabolic systems, e.g. calcium-dependent endonucleases, which split the DNA chain. Damaged DNA becomes more immunogenic, causing autoantibody production. Studies on hypoxanthine/xanthine oxidase-dependent damage to DNA structure in the presence of calcium ions identified products which may be used as oxidative DNA damage markers. These are 5,6-dihydrocytozine,4,6-diamino-5-formamidopirymidine, 2,6-diamino-4-hydoxy-5-form-amidopirymidine, 8-hydroxyadenine, 8-hydroxyguanine, and 8-hydroxy-2'-deoxyguanosine [5, 34].

Both non-radical and radical species have been demonstrated to be capable of degrading in vivo proteoglycans, which are the main components of the basic substance of connective tissue. Hyaluronic acid depolymerization caused by ROS may be characterized by glycoside bond fragmentation between monomers (between glucuronic acid and acetylglucosamine) [10, 36]. Many other extracellular matrix components (collagen, fibronectin, laminin) have been found to be degraded to low-molecular-weight peptides.

For homeostasis, extra- and intra-cellular preventive mechanisms must exist. This function is played by antioxidants, which may be regarded as those substances which, when present at low concentrations compared with those of an oxidizable substrate, will significantly delay or inhibit oxidation of that substrate $[4,6,7,11,36]$.

Oxidative stress is defined as an imbalance between oxidant and antioxidant protection. It is an important etiopathogenic factor in over 50 general diseases [5], including atherosclerosis [19] with its complications and diabetes mellitus [15, 27], for which, in turn, periodontitis is a risk factor. In this context it is interesting to assess the parameters connected with oxidative stress in periodontitis. In addition, there are few published data on the roles of free radicals and antioxidants in the pathogenesis of periodontal diseases.

The aim of this study was to determine 8-hydroxy-2'-deoxyguanosine (8-OHdG) concentrations and total antioxidant status (TAS) in peripheral and gingival blood serum of periodontitis patients. The relationships between these markers of oxidative stress and total antioxidant capacity and clinical parameters of periodontitis were examined.

\section{MATERIALS AND METHODS}

The study included 56 periodontitis patients, 33 females and 23 males, aged 18-55 years (mean: 39.7 years). All patients signed informed consent forms prior to enrollment in the study. Approval for the study was obtained from the Ethics Committee of Wrocław Medical University. The patients were enrolled in the study according to the following criteria: absence of systemic disease, lack of any sign of infection, and no use of systemic medications which could affect immuno-inflammatory reactions, microflora, or oxidative stress (especially the intake of vitamins A, C, and E). Medical histories were assessed to exclude patients with conditions in which oxidative stress played an important role. The existence of such disease (even in the past) disqualified such patients. Smokers were also excluded from this study.

Aggressive periodontitis (AgP) [23] was diagnosed in patients with rapid attachment loss (generalized GAP: periodontal pocket depth $(\mathrm{PD})>5 \mathrm{~mm}$ around at least three teeth other than the first molars and incisors, localized: PD $>5 \mathrm{~mm}$ around two teeth, one of which is a first molar, and involving no more than two teeth other than the first molar and incisors), rapid bone destruction ( $>50 \%$ bone loss at diseased sites), lack of or a weak relationship between dental plaque and the severity of gingival inflammation, and familial aggregation. Chronic periodontitis (CP) [25] was diagnosed in patients with at least 10 sites with $\mathrm{PD}>5 \mathrm{~mm}$, evidence of bone loss of more than $1 / 3$ of the root length on the last tooth per quadrant, an association between the extent of gingival inflammation and dental plaque, and association with a local predisposing factor (e.g. subgingival calculus or iatrogenic factors).

The following patients were included in the study: 26 subjects, 18 females and 8 males aged 18-45 years (mean: 31.5 years), with $\mathrm{AgP}$ and 30 subjects, 15 females and 15 males aged 35-55 years (mean: 44.9 years) with CP. The control group consisted of 25 healthy volunteers, 15 females and 10 males aged 22-50 years (mean: 33.2 years), in good general health without any signs of gingival inflammation or periodontal destruction. The periodontal status of the subjects was determined by measuring the plaque index (PI) [31], approximalraum plaque index [24], papillary bleeding index (PBI) [29], and the modified sulcular bleeding index [1] and by recording $\mathrm{PD}$. The clinical parameters are presented in Table 1. All of the clinical examinations were performed by one examiner, a periodontist who was not blinded, in the periodontology department.

Blood samples were taken in the morning between 8:00-9:30, the subjects having had no food or drink. The samples $(5 \mathrm{ml})$ were taken from the cubical vein and gingival blood (100 $\mu \mathrm{l})$ was aspirated during PBI measurement by gentle puncture of the gingival papilla at the sites of the most severe inflammation. Gingival blood was collected into heparinized glass microcapillary tubes (the same as for hematocrit test) using an Eppendorf pipette and centrifuged immediately. Peripheral blood samples were clotted for $30 \mathrm{~min}$ and centrifuged at $1000 \times \mathrm{g}$ for $10 \mathrm{~min}$. The serum samples were stored at $-80^{\circ} \mathrm{C}$ until assayed.

8-OHdG levels were measured in duplicate by a competitive ELISA kit (OXIS, Portland, OR, USA) 
Table 1. Clinical parameters in experimental groups and controls

\begin{tabular}{lccccc}
\hline Parameter & $\begin{array}{c}\text { All diseased patients } \\
(\mathrm{n}=56)\end{array}$ & $\begin{array}{c}\text { AgP } \\
(\mathrm{n}=26)\end{array}$ & $\begin{array}{c}\text { GAP } \\
(\mathrm{n}=20)\end{array}$ & $\begin{array}{c}\text { CP } \\
(\mathrm{n}=30)\end{array}$ & $\begin{array}{c}\text { Control } \\
(\mathrm{n}=25)\end{array}$ \\
\hline PI & $0.96 \pm 0.71$ & $0.91 \pm 1.12$ & $1.03 \pm 0.87$ & $1.10 \pm 0.58$ & $0.18 \pm 0.31^{1}$ \\
API & $75.2 \pm 21.3$ & $69.2 \pm 33.7$ & $79.3 \pm 23.5$ & $75.3 \pm 19.8$ & $20.3 \pm 20.5^{2}$ \\
PBI & $2.07 \pm 0.97$ & $2.11 \pm 1.45$ & $2.49 \pm 0.97$ & $1.87 \pm 0.86$ & $0.27 \pm 0.22^{3}$ \\
mSBI & $76.6 \pm 23.0$ & $73.5 \pm 35.7$ & $84.4 \pm 20.6$ & $75.5 \pm 21.6$ & $15.1 \pm 10.1^{4}$ \\
PD & $4.17 \pm 1.02$ & $4.28 \pm 1.51$ & $4.65 \pm 0.96$ & $4.02 \pm 0.84$ & $1.94 \pm 0.21^{5}$ \\
\hline
\end{tabular}

${ }^{1}$ Differences between all periodontitis group and controls are $\mathrm{p}<0.001 ;{ }^{2}$ differences between all periodontitis group and controls are $\mathrm{p}<0.001 ;{ }^{3}$ differences between all periodontitis group and controls are $\mathrm{p}<0.001 ;{ }^{4}$ differences between all periodontitis group and controls are $\mathrm{p}<0.001 ;{ }^{5}$ differences between all periodontitis group and controls are $\mathrm{p}<0.001$.

PI - plaque index, API - approximalraum plaque index, PBI - papillary bleeding index, mSBI - modified sulcular bleeding index, $\mathrm{PD}$ - periodontal pocket depth, GAP - generalized agressive periodontitis.

according to the manufacture's instructions. The sensitivity of the method was $1 \mathrm{ng} / \mathrm{ml}$. Intra- and inter-assay coefficients of variation for the procedure were 9.2 and $10.8 \%$, respectively.

TAS was evaluated with a kit supplied by Randox Laboratories Ltd. using a colorimetric method based on the reduction of ABTS radical cation generation $\left(\mathrm{ABTS}^{\mathrm{o}}\right.$ 2,2-azinobis-3-ethylbenzthiazoline sulfonate cation) by antioxidants in the serum samples. The antioxidative potential was examined by spectrophotometric evaluation of the intensity of staining after the addition of $\mathrm{ABTS}^{\circ+}$ cation radical from the patients' sera. TAS in the peripheral and gingival blood was presented in $\mathrm{mmol} / \mathrm{l}$ as an equivalent of Trolox (vitamin $\mathrm{E}$ analog). The calculation was performed relative to the factor $\mathrm{F}$, which was determined earlier:

$\mathrm{F}=[$ concentration of 6-hydroxy-2,5,7,8-tetramethylchroman $] /[\Delta \mathrm{A}$ (blank)- $\Delta \mathrm{A}(6$-hydroxy-2,5,7,8-tetramethylchroman)]

where $\Delta A=A_{2}-A_{1}, A_{1}$ being the initial absorbance and $\mathrm{A}_{2}$ the absorbance after exactly $3 \mathrm{~min}$ of reaction. $\mathrm{F}$ was used to calculate TAS:

$$
\mathrm{TAS}=\mathrm{F} \times[\Delta \mathrm{A}(\text { blank })-\Delta \mathrm{A}(\text { sample })]
$$

The intra- and inter-assay coefficients of variation were 3.7 and $4.2 \%$, respectively.

Each variable was tested by the Shapiro-Wilk test and Levene's test for homogeneity of variances. Student's $t$-test was used for a normal distribution and homogenous variances and Mann-Whitney's test for a non-normal distribution and/or non-homogenous variances. The level of significance was $\alpha=0.05$. To detect a relationship between two variables, Spearman's correlation test was conducted. The level of significance was $\alpha=0.01$.

\section{RESULTS}

An increased mean concentration of $8-\mathrm{OHdG}$ was observed in the gingival blood of both patient groups compared with the control group. A significant difference in this marker's concentration between patients with $\mathrm{AgP}$
Table 2. The concentration of $8-\mathrm{OHdG}$ in venous and gingival blood

\begin{tabular}{lcc}
\hline \multicolumn{1}{c}{$\begin{array}{c}\text { Examined } \\
\text { group }\end{array}$} & $\begin{array}{c}\text { 8-OHdG level } \\
\text { in venous blood } \\
(\mathrm{ng} / \mathrm{ml})\end{array}$ & $\begin{array}{c}\text { 8-OHdG level } \\
\text { in gingival blood } \\
(\mathrm{ng} / \mathrm{ml})\end{array}$ \\
\hline $\begin{array}{l}\text { All diseased patients } \\
(\mathrm{n}=56)\end{array}$ & $10.66 \pm 4.39$ & $17.40 \pm 7.10^{1}$ \\
AgP $(\mathrm{n}=26)$ & $11.61 \pm 3.95$ & $20.36 \pm 8.44^{2}$ \\
$\mathrm{CP}(\mathrm{n}=30)$ & $9.75 \pm 4.78$ & $14.52 \pm 4.49^{3,4}$ \\
Control $(\mathrm{n}=25)$ & $11.44 \pm 8.61$ & $10.71 \pm 4.35$ \\
\hline
\end{tabular}

${ }^{1} \mathrm{p}=0.000$ compared with the control group; ${ }^{2} \mathrm{p}=0.000 \mathrm{com}-$ pared with the control group; ${ }^{3} \mathrm{p}=0.001$ compared with the control group; ${ }^{4} \mathrm{p}=0.019$ significant difference between $\mathrm{AgP}$ and $\mathrm{CP}$.

and $\mathrm{CP}$ was noted $(\mathrm{p}=0.019)$. No statistically significant difference was observed in $8-\mathrm{OHdG}$ concentration in venous blood between the examined groups (Table 2).

Subjects with CP had a significantly decreased TAS level in gingival blood compared with the control group of healthy patients. Significantly decreased TAS level in the peripheral blood in the group of all patients compared with the control group was found. There was also a significant difference in TAS level between patients with different forms of periodontitis ( $p=0.001$; Table 3 ).

Correlation analysis in all the subjects revealed a positive correlation between TAS level in gingival blood and TAS level in venous blood $(\mathrm{p}<0.001$, $\mathrm{R}=0.37$ ). A significant positive correlation was also found between TAS levels in venous and in gingival blood in the group of all periodontitis patients $(\mathrm{p}=0.001, \mathrm{R}=0.43)$ and in those with $\mathrm{CP}(\mathrm{p}=0.009$, $\mathrm{R}=0.49$ ). Gingival and peripheral levels of $8-0 \mathrm{HdG}$ and TAS did not show any significant correlation with the clinical parameters of either patient group $(p>0.01)$.

\section{DISCUSSION}

Numerous studies have evaluated the use of gingival crevicular fluid (GCF) $[3,4,9,12,13,18,35]$ and saliva 
Table 3. TAS in serum of venous and gingival blood

\begin{tabular}{lcc}
\hline \multicolumn{1}{c}{$\begin{array}{c}\text { Examined } \\
\text { group }\end{array}$} & $\begin{array}{c}\text { TAS level } \\
\text { in venous blood } \\
(\mathrm{mmol} / \mathrm{l})\end{array}$ & $\begin{array}{c}\text { TAS level } \\
\text { in gingival blood } \\
(\mathrm{mmol} / \mathrm{l})\end{array}$ \\
\hline $\begin{array}{l}\text { All diseased patients } \\
(\mathrm{n}=56)\end{array}$ & $1.75 \pm 0.14^{1}$ & $1.85 \pm 0.16$ \\
$\mathrm{AgP}(\mathrm{n}=26)$ & $1.81 \pm 0.13^{2}$ & $1.89 \pm 0.14$ \\
$\mathrm{CP}(\mathrm{n}=30)$ & $1.68 \pm 0.13^{3,4}$ & $1.81 \pm 0.18^{5}$ \\
Control $(\mathrm{n}=25)$ & $1.94 \pm 0.12$ & $1.94 \pm 0.13$ \\
\hline
\end{tabular}

${ }^{1} \mathrm{p}=0.000$ compared with the control group; ${ }^{2} \mathrm{p}=0.000$ compared with the control group; ${ }^{3} \mathrm{p}=0.000$ compared with the control group; ${ }^{4} p=0.001$ significant differences between $\mathrm{AgP}$ and $\mathrm{CP} ;{ }^{5} \mathrm{p}=0.029$ compared with the control group.

$[7,9,11,13,26,33,34]$ to investigate the local activity of oxidative stress markers and antioxidants during periodontitis. In this investigation, gingival blood was used to achieve this aim. Levels of comparison of soluble adhesion molecules from gingival and peripheral blood during periodontitis were investigated by Krugluger et al. [22]. Concentrations of oxidative stress biomarkers in gingival blood should correlate to different stages of the local inflammation better than those in peripheral blood serum. The findings of the present study indicate significant differences in the activity of an oxidative stress marker and the antioxidant capacity in gingival and peripheral blood during $\mathrm{AgP}$ and CP. Sampling of the GCF demands the use of expensive Periotron ${ }^{\circledR} 8000$ to measure fluid volume. Besides that it is not clear if all the chemical compound is eluted from the paper strips. In the opinion of the present authors, sampling of the gingival blood is only minimally invasive and this biological fluid can be used to examine local etiopathogenic mechanisms in periodontitis.

8 -OHdG is currently used as a recognized biomarker of oxidant-induced DNA damage. Increased levels of circulating 8-OHdG have been detected in neoplasms, various forms of neurodegenerative diseases, rheumatoid arthritis, diabetes mellitus, chronic renal failure, and recombinant human erythropoietin resistance in hemodialysis patients $[2,15,20]$. In the accessible literature, only two studies were found in which $8-\mathrm{OHdG}$ concentration was determined in periodontitis patients, but in mixed saliva [33, 34].

The mean concentrations of $8-\mathrm{OHdG}$ in the gingival blood in both groups of periodontits patients were statistically higher than in the control group. The aggressive form of periodontitis was typified by the highest concentration of this nucleoside and it was significantly higher than in the CP patient group. Takane et al. [33, 34] reported significantly higher levels of $8-\mathrm{OHdG}$ in the saliva of periodontitis patients than in a control group. On this basis it may be concluded that the oxidative burst in periodontitis is so extensive that it leads to significant local DNA damage. Especially extensive oxidant-induced DNA damage exists in the most aggressive forms of periodontitis. In the present study, no signifi- cant correlation between 8 -OHdG concentrations in gingival blood and clinical data was found. Takane et al. [34] also found a lack of significant correlation between 8-OHdG levels in whole saliva and clinical parameters (bleeding on probing, pocket depth, and clinical attachment level). It was explained that this biomarker is influenced by present disease activity and not by the level of advancement of disease in the past. Furthermore, other data showed that measurement of salivary $8-\mathrm{OHdG}$ level may prove useful in identifying patients with periodontally involved teeth of hopeless prognosis [33].

Significantly decreased TAS levels in the gingival blood of CP patients compared with the control group were found. This result is in broad agreement with other studies on the local (GCF and saliva) activity of antioxidants in patients with periodontitis. Indeed, Guarnieri et al. [18] showed no differences in superoxide anion scavenging in GCF examination of CP patients and a control group. Chapple et al. [13] evaluated total antioxidant capacity of GCF in a chemiluminescence assay with luminol and with the use of Trolox. In the periodontitis patient group the characteristics of antioxidant protection indicated the possibility of decreased local TAS. In the same study the authors detected significantly decreased antioxidant capacity in stimulated mixed saliva of these patients. Kohen et al. [21] also confirmed, by a cyclic voltammetric assay, reduced activity of low-molecular-weight antioxidants in saliva. Concentrations of glutathione in GCF and TAS in the plasma or GCF of patients with $\mathrm{CP}$ were significantly lower than in controls [12]. Total antioxidant capacity of GCF and plasma from patients with $\mathrm{CP}$ was significantly lower than in healthy control samples [9]. Furthermore, Mashayekhi et al. [26] reported that the decrease in saliva antioxidant concentration in periodontitis subjects is associated with disease severity.

Studies based on the currently accepted methodology indicate that in periodontitis patients (especially in $\mathrm{CP}$ ) there may be a significantly decreased local capacity of antioxidant mechanisms. This is accompanied by increased ROS activity $[6,36]$ resulting from activated neutrophils and macrophages in the active phases of disease. The whole mouth, not only the periodontium, may be under the influence of these implications.

In the present study, positive correlation was shown between TAS level in gingival and peripheral blood of all patients and in the $\mathrm{CP}$ group. The absence of such a dependence in the control group with a healthy periodontium may indicate a connection between the local and the peripheral activity of the antioxidant systems only in periodontitis, especially in its chronic form.

Several factors are implicated in the total antioxidant system of serum and plasma regulation. Interpretation of changes in the capability to ROS inactivation by serum depends on the methodology (no essential correlation between TAS assessed in different ways). The conditions of serum storage before assay and the influence of such factors as diet, physical activity, smoking habit, type of lipid, proteins, and nucleic acid perox- 
idation are also of importance $[16,27]$. It is a very dynamic system. At the first stage of the oxidative burst in serum or plasma, an adaptive increase in antioxidant concentration is observed. The subsequent intensification of ROS generation leads to the antioxidant protective system becoming exhausted and an in vivo decrease in TAS [27]. TAS assessment only provides information about the current capability of the antioxidant mechanisms depending on the disease phase and cannot be applied in the assessment of past lesions, not to speak of future ones.

Significantly decreasing TAS level in the serum of both of the examined periodontitis patient groups compared with the control group was detected. The lowest TAS level was observed in the CP group. Sobaniec and Sobaniec-Łotowska [32], in an experimental study in rats, showed a significant decrease in serum antioxidant enzyme concentrations (glutathione peroxidase and superoxide dimutase) 21 days after ligature-induced periodontitis. Aminoguanidine treatment significantly reduced ligature-induced periodontitis in rats by reducing nitric oxide production and oxidative stress [17]. Significantly decreased Trolox-equivalent antioxidant capacity in serum was detected in children with Papillon-Lefèvre syndrome and periodontitis [8]. No differences in the concentrations of antioxidants in serum between the group with $\mathrm{CP}$ and controls were described by Chapple et al. [13]. Sculley and LangleyEvans [30] also detected no differences in antioxidant capacity between patients with periodontitis of different intensification on the basis of the ferric-reducing ability of plasma. Discrepancies among the results of the cited studies arise from the different methodologies, the absence of homogeneity of the examined groups with respect to disease intensity, and factors reflecting TAS in serum or plasma. It should be taken into consideration that a single measurement of TAS limits the observations of the present study. Prior and Cao [28] indicated that for an accurate evaluation of the dynamic changes in antioxidant concentrations in biological systems, multiple assays are required complementary to the nature of the assessment method.

Acknowledgment: This study was supported by grant no. 538 of Wrocław Medical University.

\section{REFERENCES}

1. Ainamo J. and Bay I. (1975): Problems and proposal for recording gingivitis and plaque. Int. Dent. J., 25, 229-235.

2. Akagi S., Nagake Y., Kasahara J., Sarai A., Kihara T., Morimoto H., Yano A., Nakao K., Nanba K., Ichikawa H. and Makino H. (2003): Significance of 8-hydroxy-2'-deoxyguanosine levels in patients with chronic renal failure. Nephrology, 8, 192-195.

3. Akalin F.A., Toklu E., Renda N. (2005): Analysis of superoxide dismutase activity levels in gingiva and gingival crevicular fluid in patients with chronic periodontitis and periodontally healthy controls. J. Clin. Periodontol., 32, 238-243.
4. Baltacioğlu E., Akalin F. A., Alver A., Balaban F., Ünsal M. and Karabulut E. (2006): Total antioxidant capacity dismutase activity levels in serum and gingival crevicular fluid in post-menopausal women with chronic periodontitis. J. Clin. Periodontol., 33, 385-392.

5. Bartosz G. (2003): Druga twarz tlenu. Wolne rodniki w przyrodzie. Wydawnictwo Naukowe PWN, Warszawa.

6. Battino M., Bullon P., Wilson M. and Newman H. (1999): Oxidative injury and inflammatory periodontal disease: the challenge of anti-oxidants to free radical and reactive oxygen species. Crit. Rev. Oral Biol. Med., 10, 458-476.

7. Battino M., Ferreiro M.S., Gallardo I., Newman H. and Bullon P. (2002): The antioxidant capacity of saliva. J. Clin. Periodontol., 29, 189-194.

8. Battino M., Ferreiro M. S., Quiles J. L., Bompadre S., Leone L. and Bullon P. (2003): Alterations in the oxidation products, antioxidant markers, antioxidant capacity and lipid patterns in plasma of patients affected by Papillon-Lefèvre Syndrome. Free Radic. Res., 37, 603-609.

9. Brock G. R., Butterworth C. J., Matthews J. B. and Chapple I. L. (2004): Local and systemic total antioxidant capacity in periodontitis and health. J. Clin. Periodontol., 31, 515-521.

10. Çanakçi C. F., Tatar A., Çanakçi V., Cicek Y., Oztas S. and Orbak R. (2006): New evidence of premature oxidative DNA damage: mitochondrial DNA deletion in gingival tissue of patients with periodontitis. J. Periodontol., 77, 1894-1900.

11. Chapple I. L. (1997): Reactive oxygen species and antioxidants in inflammatory diseases. J. Clin. Periodontol., 24, 287-296.

12. Chapple I. L., Brock G., Eftimiadi C. and Matthews J. B. (2002): Glutathione in gingival crevicular fluid and its relation to local antioxidant capacity in periodontal health and disease. J. Clin. Pathol., 55, 367-373.

13. Chapple I. L., Mason G. I, Garner I, Matthews J. B., Thorpe G. H., Maxwell S. R. and Whitehead T. P. (1997): Enhanced chemiluminescent assay for measuring the total antioxidant capacity of serum, saliva and crevicular fluid. Ann. Clin. Biochem., 34, 412-421.

14. Cochrane C. G. (1991): Cellular injury by oxidants. Am. J. Med., 91, 23S-30S.

15. Dandona P., Thusu K., Cook S. Snyder B., Makowski J., Armstrong D. and Nicotera T. (1996): Oxidative damage to DNA in diabetes mellitus. Lancet, 347, 444-445.

16. Di Giacomo C., Acquaviva R., Lanteri R., Licata F., Licata A. and Vanella A. (2003): Nonproteic antioxidant status in plasma of subjects with colon cancer. Exp. Biol. Med., 228, 525-528.

17. Di Paola R., Marzocco S., Mazzon E., Dattola F., Rotondo F., Britti D., De Majo M., Genovesa T. and Cuzzocrea S. (2004): Effect of aminoguanidine in ligature-induced periodontitis in rats. J. Dent. Res., 83, 343-349.

18. Guarnieri C., Zucchelli G., Bernardi F., Scheda M., Valentini A. F. and Calandriello M. (1991): Enhanced superoxide production with no change of the antioxidant activity in gingival fluid of patients with chronic adult periodontitis. Free Radic. Res. Commun., 15, 11-16.

19. Harrison D., Griendling K. K., Landmesser U., Hornig B. and Drexler H. (2003): Role of oxidative stress in atherosclerosis. Am. J. Cardiol., 91, 7A-11A.

20. Kato A., Odamaki M. and Hishida A. (2003): Blood 8-hydroxy-2'-deoxyguanosine is associated with erythropoietin resistance in haemodialysis patients. Nephrol. Dial. Transplant., 18, 931-936. 
21. Kohen R., Beit-Yannai E., Berry E. M. and Tirosh O. (1999): Overall low molecular weight antioxidant activity of biological fluids and tissues by cyclic voltammetry. Methods Enzymol., 300, 285-296.

22. Krugluger W., Nell A., Solar P., Matejka M. and BoltzNitulescu G. (1995): Influence of sE-selectin and L-selectin on the regulation of cell migration during chronic periodontitis. J. Periodontal Res., 30, 198-203.

23. Lang N. (1999): Consensus report: aggressive periodontitis. Ann. Periodontol., 4, 53.

24. Lange D. E. (1975): Die gezielte Vorbehandlung vor systematischen Parodontalbehandlung. Zahnärztl. Welt/Reform, 8, 44-47.

25. Lindhe J. (1999): Consensus report: chronic periodontitis. Ann. Periodontol., 4, 38.

26. Mashayekhi F., Aghahoseini F., Rezaie A., Zamani M. J., Khorasani R. and Abdollahi M. (2005): Alteration of cyclic nucleotides levels and oxidative stress in saliva of human subjects with periodontitis. J. Contemp. Dent. Pract., 6, 46-53.

27. Mealey B. L. (2006): Periodontal disease and diabetes. A two-way street. JADA, 137, 26S-31S.

28. Prior R. L. and Cao G. (1999): In vivo total antioxidant capacity: comparison of different analytical methods. Free Radic. Biol. Med., 27, 1173-1181.

29. Saxer U., Turconi B. and Elsasser C. (1977): Patient motivation with the papillary bleeding index. J. Prev. Dent., 4, $20-22$.
30. Sculley D. V. and Langley-Evans S. C. (2002): Salivary antioxidant and periodontal disease status. Proc. Nutr. Soc., 61, 137-143.

31. Silness J. and Löe H. (1964): Periodontal disease in pregnancy. II. Correlation between oral hygiene and periodontal condition. Acta Odontol. Scand., 22, 121-135.

32. Sobaniec H. and Sobaniec-Łotowska M. E. (2000): Morphological examinations of hard tissues of paradontium and evaluation of selected processes of lipid peroxidation in blood serum of rats in the course of experimental periodontitis. Med. Sci. Monit., 6, 875-881.

33. Takane M., Sugano N., Ezawa T., Uchiyama T. and Ito K. (2005): A marker of oxidative stress in saliva: association with periodontally-involved teeth of a hopeless prognosis. J. Oral Sci., 47, 53-57.

34. Takane M., Sugano N., Iwasaki H., Iwano Y., Shimizu N. and Ito K. (2002). New biomarker evidence of oxidative DNA damage in whole saliva from clinically healthy and periodontally diseased individuals. J. Periodontol., 73, 551-554.

35. Tsai C. C., Chen H. S., Chen S. L., Ho Y. P., Ho K. Y., Wu Y. M. and Hung C. C. (2005): Lipid peroxidation: a possible role in the induction and progression of chronic periodontitis. J. Periodontal Res., 40, 378-384.

36. Waddington R. J., Moseley R. and Embery G. (2000): Reactive oxygen species: a potential role in the pathogenesis of periodontal diseases. Oral Dis., 6, 138-151. 


\section{In Memoriam \\ Professor Feliks Milgrom \\ (1919-2007)}

Feliks Milgrom, Professor Emeritus of the University of Buffalo, State University of New York, died on September 2nd at the age of 88 in the USA.

Professor Milgrom was one of the closest students of Prof. Ludwik Hirszfeld, who was the supervisor of his doctoral thesis in 1947. Three years later he attained the rank of associate professor. After Hirszfeld's death, and on his recommendation, Milgrom became acting director of the Institute of Immunology and Experimental Therapy, Polish Academy of Sciences, in Wrocław (Poland), and then assumed the post of director of the Department of Microbiology of the Silesian University School of Medicine in Zabrze (Poland). His scientific potential is best attested by the fact that he published three papers in Nature during his time there from 1956-1957.

In 1957 he emigrated to the USA and joined the faculty of the Department of Microbiology of the University of Buffalo School of Medicine and Biomedical Sciences, where he continued his work in the fields of the immunology of transplantation and the mechanisms of autoimmunity, becoming chair of the department in 1967 and Distinguished Professor in Microbiology in 1981.

His attachment to his teacher, Prof. Hirszfeld, is underscored by articles to his memory (e.g. L. Hirszfeld: A posthumous tribute (in Polish), Pol Arch Med Wewn 1956, 11, 1643; Fundamental discoveries in immunohematology and immunogenetics by Ludwik Hirszfeld, Vox Sang 1987, 52, 149; My relations with five generations of immunologists, Transplant Proc 1999, 31, 1493; My association with Ludwik Hirszfeld, Wrocław 1945-1954, Arch Immunol Ther Exp 1998, 46, 201). He remained in contact with Poland, and in 1994 he was awarded the Gold Cross of Merit from the President of the Republic of Poland. 

The authors: Molfetta R., Peruzzi G., Santoni A. and Paolini R.

of the review entitled "Negative signals from FceRI engagement attenuate mast cell functions" published in Arch. Immunol. Ther. Exp., 2007, 55, 219-229

are expressing their apologies to the authors of the following articles:

Gilfillan A. M. and Tkaczyk C.: "Integrated signalling pathways for mast-cell activation"

published in Nat. Rev. Immunol., 2006, 6, 218-230;

Rivera J. and Gilfillan A. M.: "Molecular regulation of mast cell activation"

published in J. Allergy Clin. Immunol., 1996, 117, 1214-1225,

for not having appropriately cited and acknowledged their work. 